\title{
Correction to: Diffeomorphism groups of critical regularity
}

\author{
Sang-hyun Kim ${ }^{1}$ (D) Thomas Koberda ${ }^{2}$
}

Published online: 1 April 2020

(C) Springer-Verlag GmbH Germany, part of Springer Nature 2020

\section{Correction to: Invent. math. https://doi.org/10.1007/s00222-020-00953-y}

Due to an oversight in the Acknowledgment the grant number from Samsung Science and Technology Foundation is wrong, it should read SSTF-BA130106 and SSTF-BA1301-51.

Publisher's Note Springer Nature remains neutral with regard to jurisdictional claims in published maps and institutional affiliations.

The online version of the original article can be found under https://doi.org/10.1007/s00222020-00953-y.

$凶$ Sang-hyun Kim

skim.math@gmail.com

http://cayley.kr

Thomas Koberda

thomas.koberda@gmail.com

http://faculty.virginia.edu/Koberda

1 School of Mathematics, Korea Institute for Advanced Study, Seoul, Korea

2 Department of Mathematics, University of Virginia, Charlottesville, VA 22904-4137, USA 